\title{
Heat Conduction Problems in a Homogeneous Pipe with Inner Nonhomogeneous Coating
}

\author{
Roman Kulchytsky-Zhyhailo ${ }^{1}$, Stanisław J. Matysiak², Dariusz M. Perkowski ${ }^{{ }^{*}}$ \\ ${ }^{1}$ Department of Mechanics and Applied Computer Science, Faculty of Mechanical Engineering, Białystok University of \\ Technology, 45C Wiejska Str, Białystok 15-351, Poland \\ ${ }^{2}$ Institute of Hydrogeology and Engineering Geology, Faculty of Geology, University of Warsaw, Al. Żwirki i Wigury 93, \\ Warsaw 02-089, Poland
}

Corresponding Author Email: d.perkowski@pb.edu.pl

https://doi.org/10.18280/ijht.390103

Received: 9 September 2020

Accepted: 16 December 2020

\section{Keywords:}

temperature, heat loss, nonhomogeneous pipe, inner coating, functionally graded materials

\begin{abstract}
The paper deals with the analysis of nonhomogeneous inner coatings for a homogeneous pipe with respect of heat loss from the outer pipe surface. Two kinds of the coatings in the form of ring layers are considered: $\left(1^{\circ}\right)$ with the thermal properties changing continuously along the coating thickness (called the coating $\mathrm{A}),\left(2^{\circ}\right)$ multilayered coatings with piecewise continuous thermal properties (called the coatings B). The analysis is connected with the stationary heat conduction problems. Some special cases of the coatings A and B are investigated. The obtained analytical results and the comparison of the coatings are presented.
\end{abstract}

\section{INTRODUCTION}

Modern engineering structures require the use of materials with appropriate thermomechanical properties. This creates materials with very complex structure. Such materials include composites with functionally changing thermomechanical properties, they are so-called gradient materials. The production of such materials requires the use of advanced technologies during their production [1]. Due to the high costs of obtaining the coatings and the functionality that should be met by such materials, they require the development of some accurate modeling methods, both numerical and analytical, allowing forecasting of temperature, heat flux, displacement and stress distribution with the expected accuracy of calculations [2].

The main purpose of materials with functional gradation of properties is their use as thermal barriers Matysiak and Perkowski [3] or as layers reducing of wear at elevated temperatures [4].

Many works on the analysis of thermal stresses for various type of constructions made of functionally graded materials can be found in the literature, for example: in paper Ootao and Ishihara [5] for empty cylinders, for plates in ref. [6], and for empty balls in ref. [7]. For the analysis of thermal stresses in nonhomogeneous thermoelastic bodies with functionally graded properties, the knowledge of temperature and heat flux distributions is needed.

The analysis of heat flow in materials with functionally graded properties can be found in ref. [8-10], where the heat conductivity coefficient is described in the exponential or power form depending on the radial coordinate. In Hosseini et al. [11], the heat flow in a heterogeneous cylinder was considered, which was divided into sub-cylinders along the wall thickness of the cylinder. Whereas in Ootao [7] the heat transfer analysis for the sphere is presented, where the heat conductivity coefficient is described by a power function, here also the approach of dividing the sphere into sub-layers, within which the thermal properties of the material are constant, was applied. The approaches presented above rely on the description of a heterogeneous medium with functional gradation of properties by means of a packet of layers within which the thermal properties are constant. However, this requires the solution of an appropriate system of equations resulting meeting the conditions of heat flux continuity and temperature on the interfaces. Another approach is the analysis of heat flow using appropriate averaged methods, namely the homogenized model with microlocal parameters Matysiak [12], and Matysiak and Woźniak [13] or tolerance description [14]. In the case of approach based on homogenization with microlocal parameters, as shown in Matysiak et al. [15] and Perkowski et al. [16] it can be used for composites with a micro-periodic structure. The model homogenized with microlocal parameters has been widely applied to solve a number of issues in solid thermomechanics [17-19].

In the literature, a very widely developed method to modeling media with functional gradation of properties is the approach using direct numerical methods, including the finite element method, for instance [20], non-mesh methods [9, 21], boundary-volume method [22].

In this article, the problem of heat conduction for an infinitely long heterogeneous pipe used to the transport of heated fluid is considered. The pipe is assumed to be composed of a homogenous foundation and a nonhomogeneous insulating inner coating. The special attention is developed to examine the reduction of heat loss from its external surface achieved in the result of using of nonhomogeneous coating on the internal pipe interface.

Two kinds of the coatings in the form of ring layers are investigated. The first one is characterized by the thermal properties changing continuously along the cover thickness and it will be called the coating A. The second one consists of multilayered coatings with piecewise continuous thermal properties (called the coating B). The coating A is composed of two components: a homogeneous insulating part and a 
gradient part described by the heat conductivity coefficient in the form of an arbitrary function dependent on the radius. Two cases of choice of this function are considered: the first one is based on the one known in literature as Voigt's rule, the second one is Reuss's rule. The considered problem of heat conduction in the pipe is assumed to be stationary and one dimensional, so the exact for of solution for the temperature is found. The coating $\mathrm{B}$ is composed of $\mathrm{n}$ homogenous ring layers. In this case the conditions of ideal thermal contact between the composite components are considered. As a special case, the coating with periodic structure is investigated. The obtained results for the coatings with respect of their applications as thermal barriers are compared and remarks resulted from the realized calculations are presented.

\section{FORMULATION OF THE PROBLEM}

Consider the problem of heat conduction for an infinitely long nonhomogeneous pipe with the outer radius $R_{0}$ and the inner radius $R_{1}$. The inner surface of pipe is kept at a constant temperature different from the temperature of outer medium of the value $\theta_{0}$. The heat exchange with the outer medium is assumed to be realized according to Newton law. The considerations will be derived in the dimensionless cylindrical coordinates $(r, \varphi, z)$ concerned to the radius $R_{0}$. The distribution of temperature is assumed to be axially symmetrical and does not depend on the coordinate $z$ (in the axial direction). The nonhomogeneous pipe in its cross-section is composed of the homogeneous foundation with the coefficient of thermal conductivity $K_{0}$ and located at the region $r_{2}<r<1$, where $r_{2}=R_{2} / R_{0}$, as well as of the nonhomogeneous coating with the thickness $H=R_{2}-R_{1}$ (Figure 1). The coating takes up the region $r_{1}<r<r_{2}$, where $r_{1}=R_{1} / R_{0}$.

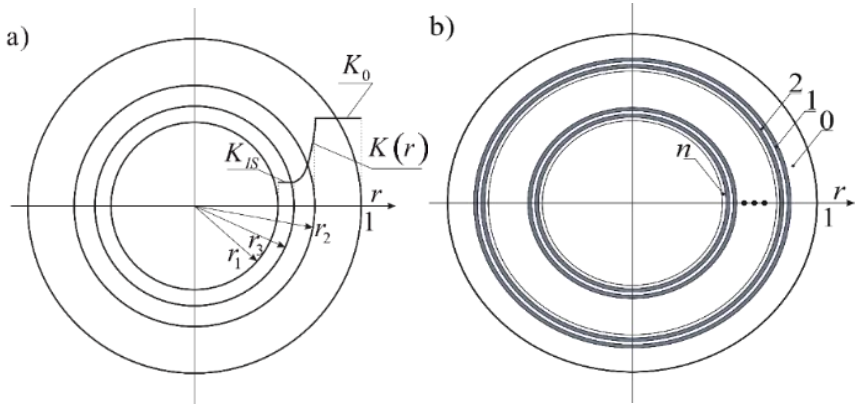

Figure 1. Scheme of considered problem

Two kinds of coatings will be considered. The coating called coating A (Figure. 1a) is composed of two components. The first component is a homogeneous insulating coating with the thermal conductivity coefficient $K_{\text {is, }}$, which contains the inner surface of pipe and it is located at the region $r_{1}<r<r_{3}$, where $r_{3}=R_{3} / R_{0}$. The second one is a gradient coating with the thermal conductivity coefficient described by the function $K(r) / K_{0}=K^{*}(r), r_{3}<r<r_{2}$. It will be assumed, that $K^{*}\left(r_{3}+0\right)=$ $K_{\text {is }} / K_{0}, K^{*}\left(r_{2}-0\right)=1$. The coating called coating B is assumed to be composed of homogeneous components with the thermal conductivity coefficients $K^{(i)}, i=1,2, \ldots, n$, which occupy the regions $r^{(i)}<r<r^{(i+1)}, r^{(1)}=r_{2}, r^{(n+1)}=r_{1}$, (Figure 1b). Moreover, it will be assumed that in the both cases of coatings (coating A and $\mathrm{B}$ ) the thermal contact between the components of pipe is ideal.

\section{MATHEMATICAL MODELS AND METHODS OF SOLUTIONS}

\section{Coating A}

The temperature field in the coating A will be obtained from the solution of following boundary-value problem:

a) the equation:

$$
\frac{d}{d r}\left(r \breve{K}(r) \frac{d T}{d r}\right)=0, r \in\left(r_{1}, r_{3}\right) \cup\left(r_{3}, r_{2}\right) \cup\left(r_{2}, 1\right)
$$

b) the boundary conditions:

- on the inner surface of pipe:

$$
T\left(r_{1}\right)=\theta_{0}
$$

- the thermal exchange on the outer surface of pipe:

$$
T^{\prime}+B i T=0, r=1
$$

- the ideal thermal contact between the components of pipe:

$$
T\left(r_{i}-0\right)=T\left(r_{i}+0\right), T^{\prime}\left(r_{i}-0\right)=T^{\prime}\left(r_{i}+0\right), i=2,3 .
$$

In the Eqns. (1)-(2) the following notations are introduced: $T$ is the function described the temperature deviation in the points of pipe from the temperature of outer medium, $T^{\prime}$ is the derivative of function $T ; B i=\alpha R_{0} / K_{0}$, where $\alpha$ is the coefficient of heat exchange between the outer surface of pipe and the outer medium,

$$
\breve{K}(r)= \begin{cases}K_{\text {is }} / K_{0}, & r_{1} \leq r \leq r_{3}, \\ K^{*}(r), & r_{3}<r<r_{2}, \\ 1, & r_{2} \leq r \leq 1 .\end{cases}
$$

Integrating twice the Eq. (1) we obtain:

$$
T(r)=C_{1} \int_{r}^{1} \frac{d x}{x \breve{K}(x)}+C_{2}, r_{1} \leq r \leq 1 .
$$

It is easy to prove, that for the arbitrary piecewise continuous function $\breve{K}(r)$ determined in the interval $\left(\mathrm{r}_{1}, 1\right)$, the Eq. (4) satisfies the conditions of ideal thermal contact (2c). The unknown parameters $C_{1}$ and $C_{2}$ are calculated using the boundary conditions (2a) and (2b):

$$
\begin{aligned}
& \frac{C_{1}}{\theta_{0}}=B i\left(1+B i \int_{r_{1}}^{1} \frac{d x}{x \breve{K}(x)}\right)^{-1}, \\
& \frac{C_{2}}{\theta_{0}}=\left(1+B i \int_{r_{1}}^{1} \frac{d x}{x \breve{K}(x)}\right)^{-1} .
\end{aligned}
$$

Introducing Eqns. (5a) and (5b) into Eq. (4), the relation determined the temperature distribution in the considered pipe can be written in the form 


$$
\frac{T(r)}{\theta_{0}}=\left(1+B i \int_{r_{1}}^{1} \frac{d x}{x \breve{K}(x)}\right)^{-1}\left(1+B i \int_{r}^{1} \frac{d x}{x \breve{K}(x)}\right), r_{1} \leq r \leq 1
$$

It can be emphasized that the obtained solution applying the conditions of ideal thermal contact between the components of pipe also in the case, when the function $\breve{K}(r)$ is a piececontinuous function in the region $\left(r_{1}, 1\right)$.

The gradient part of the coating A, $\left(r_{3}<r<r_{2}\right)$ is assumed to be of two-components composite. The first of component is the foundation material, the second one is insulating material with the heat conductivity coefficient $K_{\text {is. }}$ In the aim for the elimination of jump changes of thermal properties on the interfaces, the voluminal contribution of the insulator in the representative composite cell $V_{\text {is }}$ is changed along the coating thickness. So, the function $V_{\text {is }}(r)$ is assumed to be the linear function:

$$
V_{\text {is }}(r)=\frac{r_{2}-r}{r_{2}-r_{3}}, r_{3}<r<r_{2}
$$

For the determination of $K^{*}(r)$ in the literature the Voigt rule is often taken into calculations [23]

$$
K_{0} K^{*}(r)=K_{0}\left(1-V_{\text {is }}(r)\right)+K_{\text {is }} V_{\text {is }}(r), r_{3}<r<r_{2},
$$

Another choice is the Reuss rule [24]

$$
\frac{1}{K_{0} K^{*}(r)}=\frac{1-V_{\text {is }}(r)}{K_{0}}+\frac{V_{\text {is }}(r)}{K_{\text {is }}}, r_{3}<r<r_{2} \text {. }
$$

In the case of Voigt's model $K^{*}(r)$ is linear:

$$
\begin{aligned}
& K^{*}(r)=K_{A} r-K_{B}, r \in\left(r_{3}, r_{2}\right), \\
& K_{A}=\frac{K_{0}-K_{\text {is }}}{K_{0}\left(r_{2}-r_{3}\right)}, K_{B}=\frac{K_{0} r_{3}-K_{\text {is }} r_{2}}{K_{0}\left(r_{2}-r_{3}\right)} .
\end{aligned}
$$

whereas in the Reuss's model, $K^{*}(r)$ is the hyperbolic function:

$$
\begin{aligned}
& K^{*}(r)=\frac{1}{K_{B}-K_{A} r}, r \in\left(r_{3}, r_{2}\right), \\
& K_{A}=\frac{K_{0}-K_{\mathrm{is}}}{K_{\text {is }}\left(r_{2}-r_{3}\right)}, K_{B}=\frac{K_{0} r_{2}-K_{\mathrm{is}} r_{3}}{K_{\text {is }}\left(r_{2}-r_{3}\right)} .
\end{aligned}
$$

Substituting Eqns. (9a), (9b) into (6) it follows that

$$
\begin{aligned}
& \frac{T(r)}{\theta_{0}}= \\
& = \begin{cases}T_{D}^{-1}\left(1+B i\left(K_{0} K_{\mathrm{is}}^{-1} \ln \left(r_{3} / r\right)+T_{C}\left(r_{3}\right)\right)\right), r_{1} \leq r<r_{3}, \\
T_{D}^{-1}\left(1+B i T_{C}(r)\right), & r_{3} \leq r<r_{2}, \\
T_{D}^{-1}(1-B i \ln (r)), & r_{2} \leq r \leq 1,\end{cases}
\end{aligned}
$$

where

$$
\begin{gathered}
T_{C}(r)=K_{B}^{-1} \ln \frac{r\left(K_{A} r_{2}-K_{B}\right)}{r_{2}\left(K_{A} r-K_{B}\right)}-\ln \left(r_{2}\right), \text { (the Voigt's } \\
\text { model }) ;
\end{gathered}
$$

or

$$
\begin{gathered}
T_{C}(r)=K_{B} \ln \left(r_{2} / r\right)-K_{A}\left(r_{2}-r\right)-\ln \left(r_{2}\right), \text { (the } \\
\text { Reuss's model) }
\end{gathered}
$$

and

$$
T_{D}=1+B i\left(K_{0} K_{\text {is }}^{-1} \ln \left(r_{3} / r_{1}\right)+T_{C}\left(r_{3}\right)\right)
$$

Taking into considerations in Eqns. (10)-(12) that $r_{3}=r_{1}$, the relations for the coating with the heat conductivity coefficient changing along the all thickness of A coating are obtained (the coating $\mathbf{A}^{\prime}$ ):

$$
\frac{T(r)}{\theta_{0}}= \begin{cases}T_{D}^{-1}\left(1+B i T_{C}(r)\right), & r_{1} \leq r<r_{2} \\ T_{D}^{-1}(1-B i \ln (r)), & r_{2} \leq r \leq 1\end{cases}
$$

Passing in the equation (6) to the limit $r_{3} \rightarrow r_{2}$, the temperature in the case of homogeneous coating is given (coating $\mathbf{A}^{\prime \prime}$ ):

$$
\frac{T(r)}{\theta_{0}}=\left\{\begin{array}{l}
T_{D}^{-1}\left(1+B i\left(K_{0} K_{\mathrm{is}}^{-1} \ln \left(r_{2} / r\right)-\ln \left(r_{2}\right)\right)\right), r_{1} \leq r<r_{2}, \\
T_{D}^{-1}(1-B i \ln (r)), \quad r_{2} \leq r \leq 1 .
\end{array}\right.
$$

where

$$
T_{D}=1+B i\left(K_{0} K_{\mathrm{is}}^{-1} \ln \left(r_{2} / r_{1}\right)-\ln \left(r_{2}\right)\right)
$$

The intensity of heat loss will be estimated using the dimensionless parameter $Q$ calculated on the basis of the heat flux $q$ flowing through the outer pipe surface:

$$
Q=\frac{R_{0} q}{K_{0} \theta_{0}}=-\frac{T^{\prime}(1)}{\theta_{0}}=\frac{B i}{T_{D}} .
$$

The values of parameter $Q$ will be compared with the values of parameter $Q_{0}$, which describes the heat loss on the outer surface of the homogeneous pipe made from the material of foundation. Taking in the Eqns. (14), (15) that $K_{\text {is }}=K_{0}$ it follows what

$$
Q_{0}=\frac{B i}{1-B i \ln \left(r_{1}\right)}
$$

\section{Coating B}

For the coating B being multilayered coating composed of $n$ homogeneous annulus layers, the distribution of temperature will be determined from the equations:

$$
\frac{d}{d r}\left(r \frac{d T_{i}}{d r}\right)=0, r \in\left(r^{(i)}, r^{(i+1)}\right), i=0,1, \ldots, n
$$

the boundary conditions:

- on the inner surface of pipe:

$$
T_{n}\left(r^{(n+1)}\right)=\theta_{0}
$$




$$
T_{0}^{\prime}+B i T_{0}=0, r=r^{(0)}
$$

and conditions of ideal thermal contact between the pipe components:

$$
\begin{aligned}
& T_{i-1}\left(r^{(i)}-0\right)=T_{i}\left(r^{(i)}+0\right) \\
& K^{(i-1)} T_{i-1}^{\prime}\left(r^{(i)}-0\right)=K^{(i)} T_{i}^{\prime}\left(r^{(i)}+0\right), i=1,2, \ldots, n .
\end{aligned}
$$

In the Eqns. (17)-(20) the index $i=0$ determines parameters and state quantities in the homogeneous foundation, the indexes $i=1, \ldots, n$ are connected with the particular ring layers of coating, the numbers of the ring layers are introduced as in Figure 1b: $r^{(0)}=1 ; K^{(0)}=K_{0}$. Integrating the Eq. (17), the temperature function in $i$-th component of pipe, $i=0,1, \ldots, n$, can be written in the form:

$$
\begin{aligned}
& T_{i}(r) / \theta_{0}=t_{2 i-1}+t_{2 i} \ln \left(r / r^{(i)}\right), \\
& r^{(i)} \leq r \leq r^{(i+1)}, i=0,1, \ldots, n .
\end{aligned}
$$

The unknown parameters $t_{i}, i=-1,0,1, \ldots, 2 n$, in the Eq. (21) can be calculated by applying the boundary conditions (18)-(20). It gives the following system of equations:

$$
\begin{gathered}
t_{-1}+B i^{-1} t_{0}=0, \\
t_{2 i-3}+t_{2 i-2} \ln \left(r^{(i)} / r^{(i-1)}\right)-t_{2 i-1}=0, i=1,2, \ldots, n, \\
K^{(i-1)} t_{2 i-2}-K^{(i)} t_{2 i}=0, i=1,2, \ldots, n, \\
t_{2 n-1}+t_{2 n} \ln \left(r^{(n+1)} / r^{(n)}\right)=1 .
\end{gathered}
$$

The heat loss from the outer surface of pipe with the multilayer coating B is equal to $Q=t_{0}$.

Next, three kinds of multilayered coatings will be considered. The first one called the coating $\mathbf{B}^{\prime}$ is assumed to be composed of $n=m+1$ components. The component with the number of $m+1$ is insulating coating with the heat conductivity coefficient $K_{\text {is. }}$ The remaining components $(i=1$, $2, \ldots, m)$ are chosen with the aim for an elimination of the influence of jump changes of the thermal properties along the coating thickness. The model B' is an alternative to the coating A with mathematical indirect gradient coating. Taking into account, that

$$
K_{0}^{-1} K^{(i)}=\frac{1}{r^{(i)}-r^{(i+1)}} \int_{r^{(i+1)}}^{r^{(i)}} K^{*}(x) d x
$$

the influence of the coating $\mathrm{B}^{\prime}$ can be compared with the adequately chosen coating $\mathrm{A}$. If the coating $\mathrm{A}$ is described by the Voigt's model it follows that

$$
2 K_{0}^{-1} K^{(i)}=K_{A}\left(r^{(i)}+r^{(i+1)}\right)-2 K_{B},
$$

The adequate equation for the Reuss's model has the form:

$$
K_{0}^{-1} K^{(i)}=\frac{1}{K_{A}\left(r^{(i)}-r^{(i+1)}\right)} \ln \left(\frac{K_{B}-K_{A} r^{(i+1)}}{K_{B}-K_{A} r^{(i)}}\right) .
$$

The second kind of coating B, which will be investigated, is the coating with the periodic structure (called as the coating $\left.\mathbf{B}^{*}\right)$. The periodicity cell with the dimensionless thickness $\delta=$ $\left(r_{2}-r_{1}\right) / m$ ( $m$ is a number of periodicity cell), is composed of two ring layers with the heat conductivity coefficients $K_{\text {is }}$ and $K_{0}$, and the thicknesses $\delta_{1}=\eta \delta$ i $\delta_{2}=(1-\eta) \delta$, where the parameter $\eta \in(0,1)$ is constant along the coating thickness. It can be emphasized, that the volume fraction of insulator in the periodicity cell $V_{\text {is }}$ equals to the parameter $\eta$ only in the case of $m \rightarrow \infty$.

The solution for the coating $\mathrm{B}^{*}$ will be compared with the solution of problem, in which the nonhomogeneous coating $\mathrm{B}^{*}$ is replaced by the homogenized coating $B_{\text {hom }}$ with the thermal properties determined by using the homogenization method with microlocal parameters [25]. The boundary value problem for the replacement coating $\mathrm{B}_{\text {hom }}$ has the following form:

$$
\frac{d}{d r}\left(r \frac{d T}{d r}\right)=0, r \in\left(r_{1}, r_{2}\right) \cup\left(r_{2}, 1\right),
$$

the boundary conditions:

a) on the inner surface of pipe is the condition (2a),

b) on the outer surface of pipe is the condition (2b),

c) the conditions of ideal thermal contact between the foundation and the coating:

$$
T\left(r_{2}-0\right)=T\left(r_{2}+0\right), K_{\mathrm{hom}} T^{\prime}\left(r_{2}-0\right)=K_{0} T^{\prime}\left(r_{2}+0\right),
$$

where

$$
K_{\mathrm{hom}}=\frac{K_{0} K_{\mathrm{is}}}{\eta K_{0}+(1-\eta) K_{\mathrm{is}}}
$$

According to the homogenized model with microlocal petameters, the function $T(r), r \in\left(r_{1}, r_{2}\right)$ describes the macrotemperature in the points of coating, so $T(r)$ is the averaging temperature with respect of the periodicity cell. The solution of the above problem is given in Eqns. (14) and (15), in which the parameter $K_{\text {is }}$ is replaced by the parameter $K_{\text {hom }}$.

The third kind of the coating $B$ is the coating denoted by B", which is composed of $n=2 m+1$ components. The last $(2 m+1)$-th component like the last component in the model $\mathrm{B}^{\prime}$ is the insulating material with the heat conductivity coefficient $K_{\text {is }}$ and it is located in the region $r_{1}<r<r_{3}$. The remaining components form a quasi-periodic structure composed of $m$ cells with the same dimensionless thickness $\delta$ $=\left(r_{2}-r_{3}\right) / m$. These cells are located in the regions $\left(r^{(2 i+1)}, r^{(2 i-}\right.$ 1)), where $r^{(2 i-1)}=r_{2}-(i-1) \delta, i=1,2, \ldots, m+1$. Every cell is composed of two ring layers with the heat conductivity coefficients $K_{\text {is }}$ and $K_{0}$. The coating B" is introduced with the aim of modeling of the insulating material and the foundation with the smoothly passing from the thermal properties of foundation to the thermal properties of insulator. For this reason the volume participation of insulator with the heat conductivity coefficient $K_{\text {is }}$ in $i$-th representative cell $V_{i s}^{*,(i)}, i=$ $1,2, \ldots, m$ increases together with the increase of its number from the value near 0 to the value near 1 . To compare the 
influence of the coating B" with the influence of the coating A it is assumed, that the parameter $V_{i s}^{*,(i)}$ is equal to the mean value of function $V_{\text {is }}(r)$ described in Eq. (7) in the region $\left(r^{(2 i+1)}\right.$, $\left.r^{(2 i-1)}\right)$, so:

$$
\begin{aligned}
& V_{\mathrm{is}}^{*,(i)}=\frac{1}{r^{(2 i-1)}-r^{(2 i+1)}} \int_{r^{(2 i+1)}}^{r^{(2 i-1)}} V_{\mathrm{is}}(x) d x= \\
& =\frac{2 r_{2}-r^{(2 i+1)}-r^{(2 i-1)}}{2\left(r_{2}-r_{3}\right)}=\frac{2 i-1}{2 m}, i=1,2, \ldots, m .
\end{aligned}
$$

If the insulating ring layer is the first layer of representative cell then:

$$
\begin{aligned}
& \left(r^{(2 i)}\right)^{2}=\frac{2 m+1-2 i}{2 m}\left(r^{(2 i-1)}\right)^{2}+\frac{2 i-1}{2 m}\left(r^{(2 i+1)}\right)^{2}, \\
& i=1,2, \ldots, m .
\end{aligned}
$$

Instead, when it is the second layer of representative cell then

$$
\begin{gathered}
\left(r^{(2 i)}\right)^{2}=\frac{2 i-1}{2 m}\left(r^{(2 i-1)}\right)^{2}+\frac{2 m+1-2 i}{2 m}\left(r^{(2 i+1)}\right)^{2}, \\
i=1,2, \ldots, m .
\end{gathered}
$$

\section{ANALYSIS OF THE RESULTS}

The analysis of obtained relations shows, that the solution of the problem in the case of coating A depends on the function $K^{*}(r)$ and five dimensionless parameters: three ratios between the radiuses od surfaces of the component regions: $R_{1} / R_{0}, R_{2} / R_{0}$ and $R_{3} / R_{0}$; the ratio between the heat conductivity coefficient of insulator and foundation $K_{\mathrm{is}} / K_{0}$, and the parameter $B i$ described the level of heat exchange between the pipe and the outer medium. The solutions of the considered kinds of the coating $\mathrm{B}$ depends also on the number $n$ ring layers in the coating, as well as on the parameters $\eta$ in the case of multilayered coating with periodic structure $\mathrm{B}^{*}$. In the aim of decreasing of the number of analysed parameters and the range of its values, the following assumptions are taken into considerations:

$1^{0}$ ) the ratio between the pipe thickness and its radius is 0.1 , so $R_{1} / R_{0}=0.9$;

$2^{0}$ ) the thickness of insulating homogeneous coating is the same as the thickness of gradient coating, so $2 R_{3}=R_{1}+R_{2}$;

$3^{0}$ ) the thickness of coating is smaller than the half of pipe thickness;

$4^{0}$ ) the parameter $B i \geq 1$.

The distributions of temperature along the pipe thickness are shown in Figure 2a, and the relations between the intensity of thermal loss from the outer pipe surface and the parameter $B i$ are presented in Figure $2 \mathrm{~b}$, which are obtained for two alternative mathematical models of coatings, namely for the coating A and B'. The continuous lines in these figures describe the influence of coating $A$, the rhombuses describe the influence of coating B. The black lines are obtained for the case of averaging heat conductivity coefficient given by Reuss's relation (Eq. (8a)), the grey lines for the Voigt's relation (Eq. (8b)). The broken lines in Figure $2 \mathrm{~b}$ and some next figures show the limit case $B i \rightarrow \infty$, which is adequate to the assumption, that the temperature on the outer pipe surface is equal to the temperature of outer medium. a)

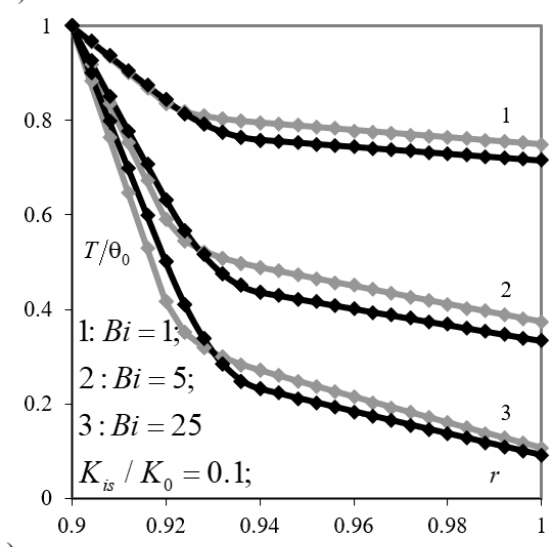

b)

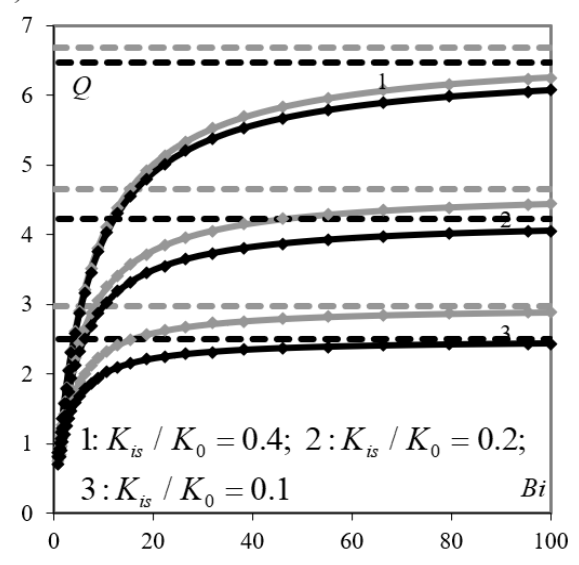

Figure 2. (a) The temperature distribution along the pipe thickness, (b) The influence intensity of heat loss from the outer pipe surface for coating A and B' $(n=11)$; the continuous lines for the coating A; rhombuses for the coating B'; the black lines and rhombuses for Reuss's model; the gray lines and rhombuses for Voigt's model; the broken lines for $B i \rightarrow \infty ; R_{1} / R_{0}=0.9 ; R_{2} / R_{0}=0.94 ; R_{3} / R_{0}=0.92$

The temperature on the outer pipe surface decreases together with an increase of the parameter $B i$; the heat loss from the outer pipe surface increases together with increase of the parameter $B i$ and decreases together with decrease of the heat conductivity coefficient of insulator. The solutions of the considered problems, together with an increase of the parameters $B i$ tend asymptotically to the limit value obtained under assumption that the temperature on the outer pipe surface is equal to the temperature of outer medium. The differences between the solutions obtained on the basis of Reuss's and Voigt's relations increase together with a decrease of the parameter $K_{\text {is }} / K_{0}$. The calculations also show that between the solutions obtained for two different manners of averaging of the thermal properties there are full convergence qualitative and quantitative. Even for a relatively small number of ring layers $(m=10)$, on which the gradient coating was divided, the deviation for the calculations of the intensity heat loss from the outer pipe surface is not greater than $0.25 \%$. It is shown that small differences between the distributions of heat conductivity coefficient along the coating, which appears in the coatings A and B', have an insignificant influence on the problem solutions and they can be omitted during an analysis of the problems.

The comparison of relations between the parameter $Q$ and the parameter $B i$ in the problem connected with coating A with averaging thermal properties and the problem for the multilayered coating B', which modelled bedding of the 
insulator ring layers in the indirect gradient coating. The black and grey lines in the figure are described as in the Figure $2 b$. The rhombuses present the influence of coating B',

a)

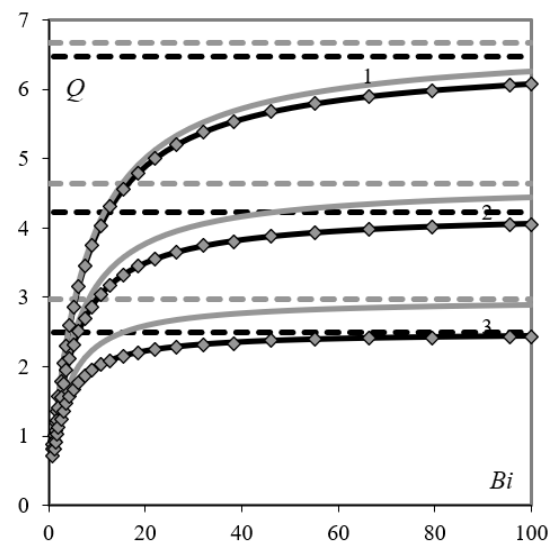

b)

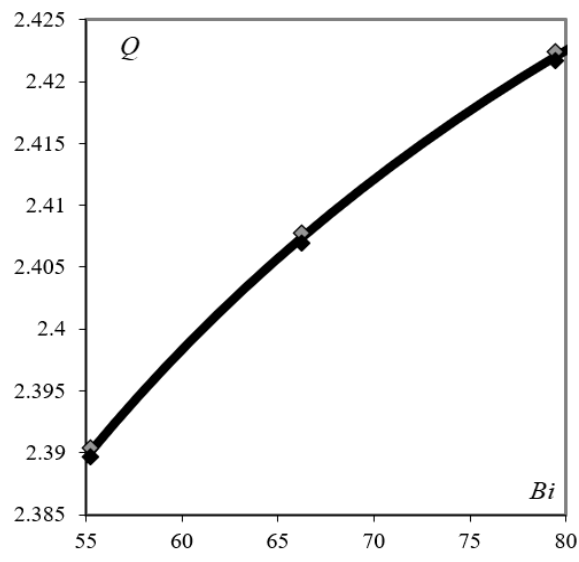

Figure 3. The dependence of heat loss intensity from the outer pipe surface with respect on the parameter $B i$ in the problems of coating A or coating B', $(n=21)$; the continuous lines are adequate for the coating $\mathrm{A}$, the rhombuses for the coating B"; the black lines for Reuss's model, the grey lines for Voigt's model; the broken lines for $B i \rightarrow \infty ; R_{1} / R_{0}=0.9$;

$$
R_{2} / R_{0}=0.94 ; R_{3} / R_{0}=0.92 ; \text { line } 1-K_{\text {is }} / K_{0}=0.4 \text {; line } 2-
$$

$$
K_{\text {is }} / K_{0}=0.2 ; 3-K_{\text {is }} / K_{0}=0.1
$$

On the basis of Figure 3a it can be inferred, that averaging of thermal properties using the Reuss model, the obtained results are complete according with the results for the structure of coating B'. Whereas, completely analogous to using of Voigt's model for calculations of heat loss gives great results since 3 to $20 \%$ in dependences of values of heat conductivity coefficient of the insulator (it is assumed that $0.1 K_{0} \leq K_{\text {is }} \leq$ $0.4 K_{0}$ ). It seems worthy emphasizing, that the solution of the problem for the coating with averaging thermal properties is independent for the sequence of layer arrangement in the representative cell of the indirect gradient coating. If the coating is taken into account (coating B') this difference is observed. However, the difference is such small, that is not visible in Figure 3a. For this reason, the some fragment of the Figure $3 \mathrm{a}$ (the fragment of curve 3 ) is shown in increasing in Figure $3 b$. The grey rhombuses in Figure $3 b$ are adequate to the structure of coating B", in which the insulating in layer is the first in the representative cell, so one of the insulating layer is located on the interface between the coating and the foundation. Whereas, the black rhombuses describe the coating structure B', in which the insulating layer is the second in the representative cell. The relative difference between the grey and black rhombuses in Figure $3 \mathrm{~b}$ is not greater than $0.04 \%$. As it is seen form Figure $3 \mathrm{~b}$, the value of parameter $Q$ in the coating A is located between the values of parameters $Q$ in the coating B" calculated for different manner of layers localization in the representative cell.

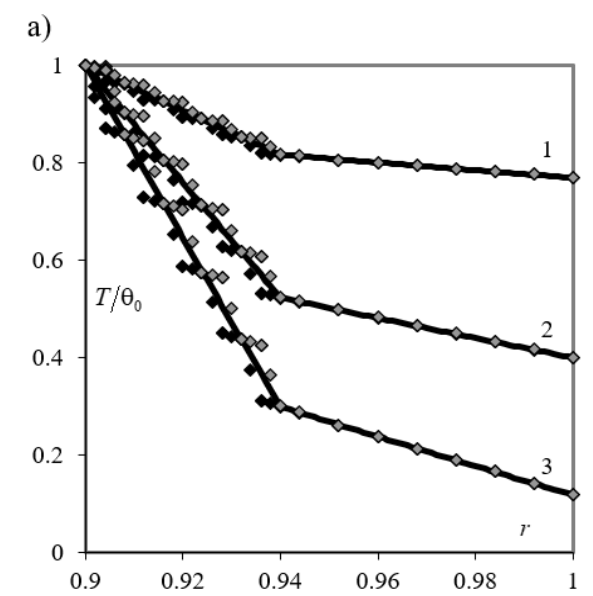

b)

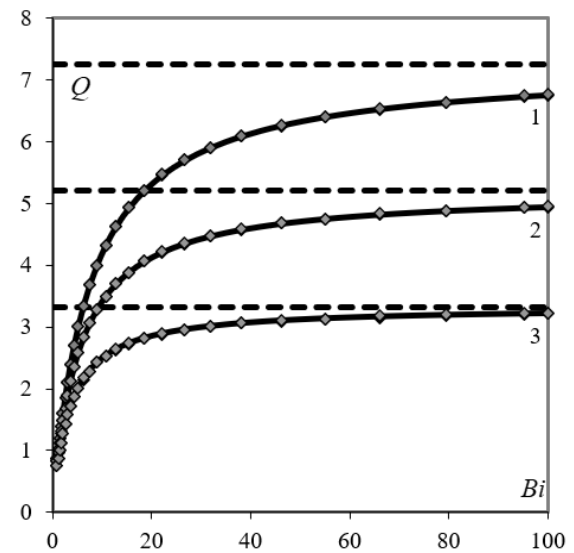

c)

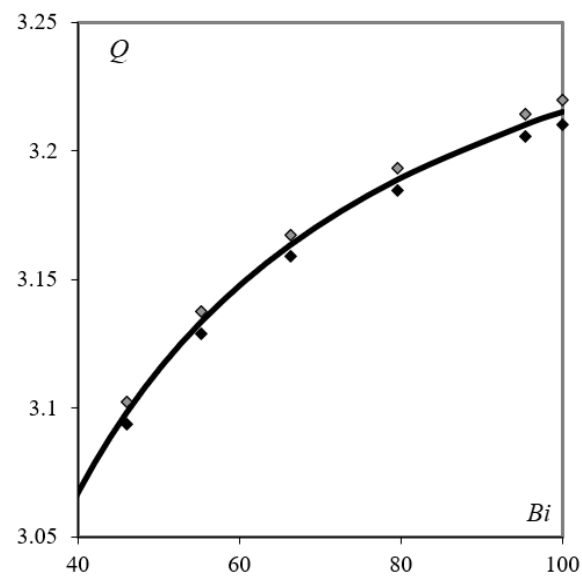

Figure 4. The distribution of temperature along the pipe thickness; (Figure 4a) and the relation of heat loss intensity with respect on the parameter $B i$; (Figure $4 \mathrm{~b}$ and $4 \mathrm{c}$ ) in the problems of multilayered coating with the periodic structure

$\mathrm{B}^{*}(n=10)$ and adequate replacing coating $\mathrm{B}_{\text {hom: }}$ the continuous lines are for the coating $\mathrm{B}_{\text {hom; }}$ rhombuses for $\mathrm{B}^{*}$; the broken lines for the case of $B i \rightarrow \infty ; R_{1} / R_{0}=0.9 ; R_{2} / R_{0}=$ $0.94 ; \eta=0.5$; in Figure $4 \mathrm{a}: K_{\mathrm{is}} / K_{0}=0.1$; the curve 1 for $B i=$ 1 ; the curve 2 for $B i=5 ; 3$ for $B i=25$; in Figure $4 \mathrm{~b}$ : the curve 1 for $K_{\text {is }} / K_{0}=0.4$; the curve 2 for $K_{\text {is }} / K_{0}=0.2$; the curve 3 for $K_{\text {is }} / K_{0}=0.1$; in Figure $4 \mathrm{c}$ for $K_{\text {is }} / K_{0}=0.1$ 
a)

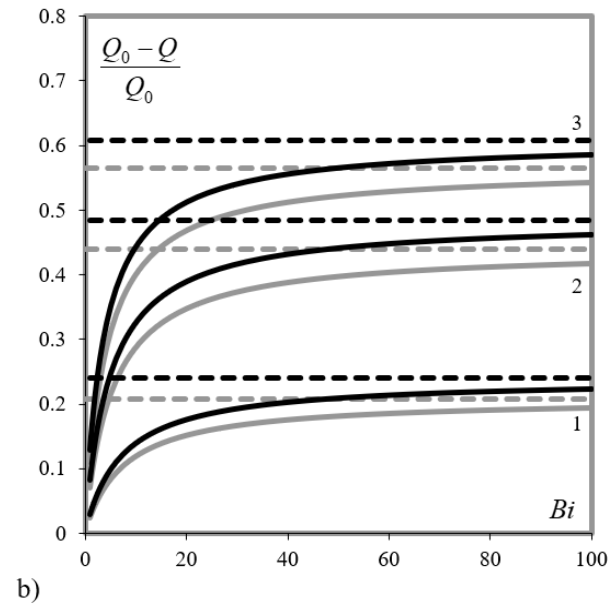

b)

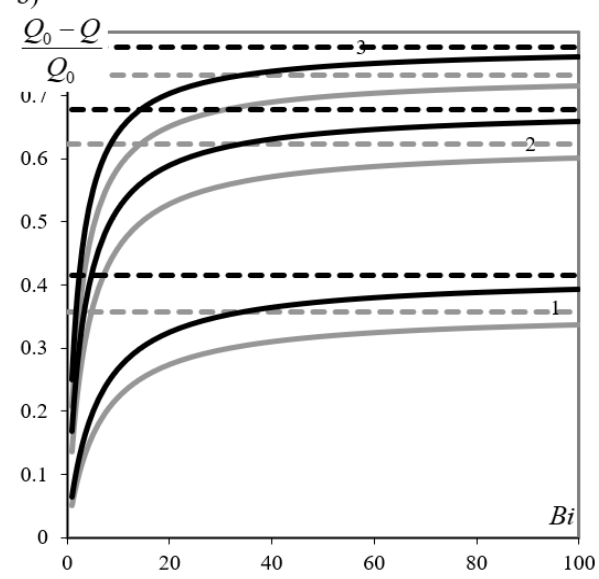

Figure 5. The relations of heat loss with respect on the parameter Bi for the coating A: the black lines for Reuss's model; the grey lines for Voigt's model; the broken lines for $B i \rightarrow \infty ; R_{1} / R_{0}=0.9 ; 2 R_{3}=R_{1}+R_{2}$; Figure $5 \mathrm{a}$ : for $K_{\text {is }} / K_{0}=$ 0.2 ; Figure $5 \mathrm{~b}$ : for $K_{\mathrm{is}} / K_{0}=0.1$; the curve 1 for $R_{2} / R_{0}=0.91$; the curve 2 for $R_{2} / R_{0}=0.93$; the curve 3 for $R_{2} / R_{0}=0.95$

The construction ally simpler kind of coating in the comparison with the coating B", is the multilayered coating $\mathrm{B}^{*}$ with periodic structure. In this coating, the representative cell is not changed along the thickness of coating, so it is the periodicity cell. The properties of solution for the coating $\mathrm{B}^{*}$ and the solution the problem, in which the coating $\mathrm{B}^{*}$ is replaced by the coating $B_{\text {hom }}$ are presented in Figure 4 . Figure $4 \mathrm{a}$ shows the temperature distribution along the coating thickness; Figures $4 \mathrm{~b}$ and $4 \mathrm{c}$ present the dependence of the parameter $Q$ with respect to the parameter $B i$. The continuous lines describe the results for the coating $B_{\mathrm{hom}}$. This solution is independent of the sequences of ring layers in the periodicity cell. Whereas, the solution of the problem for coating $\mathrm{B}^{*}$, similarly as in the case of coating B', describes such dependence. Similarly as in Figure 3b, the grey rhombuses present the case, when the ring insulator layer is the first layer in the periodicity cell and the black rhombuses, when it is the second layer. The influence of the sequence of ring layers in the case of calculations of temperature in the foundation as well as the parameter $Q$ is such small, that the black and grey rhombuses in Figures $4 \mathrm{a}$ and $4 \mathrm{~b}$ covers. For this reason, similarly as in Figure 3, the fragment of Figure 4b, (namely the fragment of curve 3 ) in increase in shown in Figure 4c. The relative difference between the grey and black rhombuses given in Figure $4 \mathrm{c}$ is near $0.25 \%$.

From Figures 4 it follows that the calculated values for the coating $B_{\text {hom }}$ are located between the adequate values obtained for the nonhomogeneous coating $\mathrm{B}^{*}$ and calculated for two different sequences of bedding of the insulator ring layer in the periodicity cell.

The calculation of temperature in the foundation and the parameter $Q$ can be replaced by the coating number $B_{\text {hom }}$ even for small number of cells $(m=5)$. The largest differences between the temperature distributions obtained within the framework of both coating are seen in the coating. The calculations show that the double increase of layer number in the coating caused the double decrease of these differences.
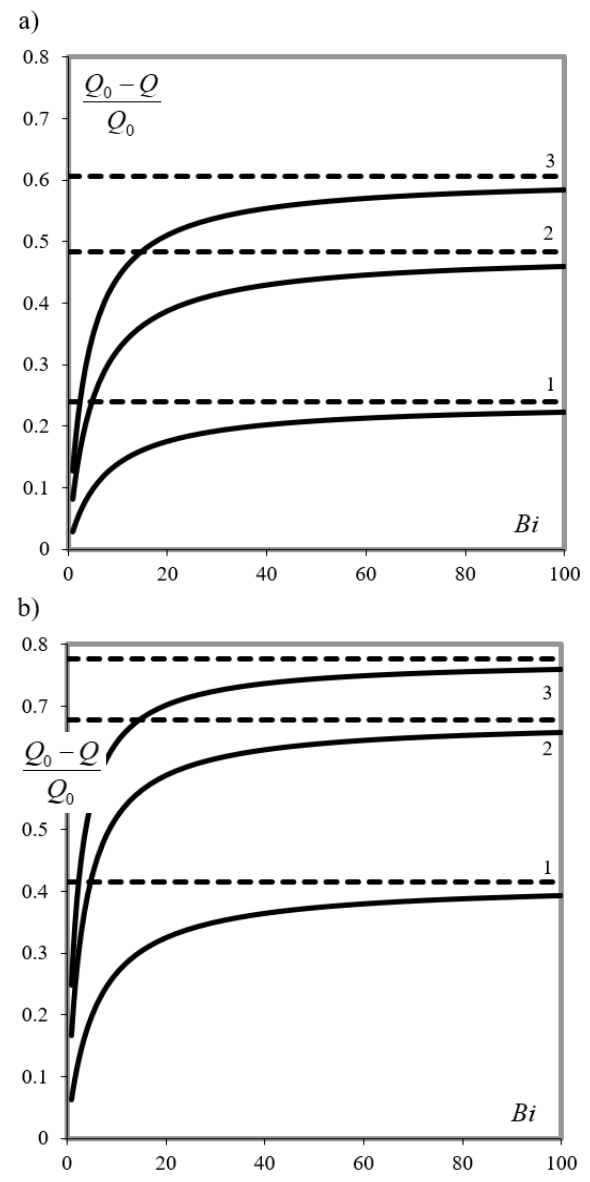

Figure 6. The relation of heat loss with respect on the parameter $\mathrm{Bi}$ for the coating $\mathrm{B}_{\text {hom }}$ : the broken lines for $\mathrm{Bi} \rightarrow$ $\infty ; R_{1} / R_{0}=0.9 ; \eta=0.75$; Figure $6 \mathrm{a}$ : for $K_{\text {is }} / K_{0}=0.2$; Figure 6b: for $K_{\text {is }} / K_{0}=0.1$; the curve 1 for $R_{2} / R_{0}=0.91$; the curve 2 for $R_{2} / R_{0}=0.93$; the curve 3 for $R_{2} / R_{0}=0.95$

Figures 5 and 6 describe the reduction of heat loss from the outer pipe surface, which is obtained by the nonhomogeneous insulating coatings on its inner interface considered in this paper. The calculations were performed for three coating thickness, two values of heat conductivity coefficient and several values of the parameters Bi. As it was expected, together with an increase of coating thickness or a decrease of the coefficient $K_{\text {is }} / K_{0}$ the level of heat loss reduction increases. This level increases also together with an increase of the parameter Bi. Calculating in the coating $\mathrm{A}$ the heat conductivity coefficient according with the Voigt's method, the values are smaller from the adequate values obtained by using the Reuss's method. The difference is equal from $5 \%$ to $15 \%$ in dependences on the parameters $B i, K_{\text {is }} / K_{0}$ and $R_{2} / R_{0}$. Calculating the parameter $\left(Q_{0}-Q\right) / Q_{0}$, the coating $\mathrm{B}^{*}$ was replaced by the coating $B_{\text {hom. }}$. For the purpose of the heat loss 
reductions, which rises by using the coating $\mathrm{A}$ and $\mathrm{B}_{\text {hom, the }}$ parameter $\eta$ is chosen $(\eta=0.75)$ in such manner, that the volume content of insulator in the both coating is the same. From Figures 5 and 6 it follows, that the heat reduction caused by the coating $\mathrm{B}^{*}$ is comparable to the heat reduction in the case of coating $\mathrm{A}$, in which the heat conductivity coefficient was calculated according with Reuss's method.

\section{CONCLUSIONS}

In the paper the methods of mathematical description of nonhomogeneous insulating coating on the inner pipe interface, which are applied in the aim of heat reduction from its outer surface, are presented. The mathematical models connected with the coating A and B' correspond to two alternative approaches of averaging of thermal properties of the representative cell. In both cases an information about the physical structure of cell is unknown. Partly it covers up in the manner of definition of the function $K^{*}(r)$. The calculations showed, that local differences in the description of thermal properties of the coatings A and B' do not contribute substantial differences in the temperature or heat flux distributions. It permits to suggest that both the mathematical model A and the mathematical model B' adequate by describe the nonhomogeneous insulating coating. The choice of model depends on efficient of mathematical method applied to solving of adequate problem. The model A is particularly effective, when an analytical solution of considered problem can be obtained. However, during solving more complicate problems, differential equations with functional coefficients, which are specific for the coating A, can make the algorism of solution difficult. In this case, more effective approach can be the mathematical model of the coating B'.

In the coating $A$, as well as in the coating $B^{\prime}$ the fundamental significance has an approach to constructing of the form of function $K^{*}(r)$. This function should reflect the structure of representative cell of nonhomogeneous gradient coating. In this paper the multilayered coating B" is considered, in which one of the simplest manner of insulator composition, in the gradient indirect coating, which assures transition from the thermal properties of foundation to the thermal properties of insulator. The calculations proved, that this coating can be replaced by the coating $A$, in which the heat conductivity coefficient in the direction normal to the layering is calculated by using Reuss's relation.

In the paper the multilayered coating with periodic structure, which the representative cell did not change along the coating thickness, so it is the periodicity cell. It is known, that in the case when the coating is composed of sufficiently large number of cells, this coating can be replaced by a homogenous coating, which the thermal properties are determined by using of homogenization method. In the paper it is shown, that the homogenization method with microlocal parameters [25] is very effective during calculations of temperature in the foundation and the heat loss from the outer pipe surface even in the case of small number (for $n=5$ ) of repeating periodicity cells. It should be emphasized that the equation to calculation of the heat conductivity coefficient in the normal direction to the layering for replacement homogenous coating given by equation (27) is consistent with the Reuss's relation given in equation (8b), which is effective in the description of the gradient coating B". It allows to suggest, that for the determination of function described the properties of gradient coating can be applied adequate relations for replacement homogenous coating obtained using the homogenization method.

\section{ACKNOWLEDGMENT}

This investigation was supported under the program of the Ministry of Science and Higher Education under the name "Regional Initiative of Excellence" in 2019-2022 (project number 011/RID/2018/19; financing amount: 12 million PLN).

\section{REFERENCES}

[1] Shigeyuki, S. (2013). Handbook of Advanced Ceramics. Elsevier, Academic Press.

[2] Celebi, K., Yarımpabuc, D., Keles, I. (2017). A novel approach to thermal and mechanical stresses in a FGM cylinder with exponentially-varying properties. Journal of Theoretical and Applied Mechanics, 55(1): 343-351. https://doi.org/10.15632/jtam-pl.55.1.343

[3] Matysiak, S., Perkowski, D. (2016). On heat conduction problems in a composite half-space with a nonhomogeneous coating. Heat Transfer Research, 47(12): 1141-1155. https://doi.org/10.1615/HeatTransRes.2016013425

[4] Hernik, S. (2016). Wear resistance of piston sleeve made of layered material structure: MMC A356R, antiabrasion layer and FGM interface. Acta Mechanica et Automatica, 10(3): 207-212. https://doi.org/10.1515/ama-2016-0031

[5] Ootao, Y., Ishihara, M. (2014). Transient thermoelastic analysis for a multilayered hollow cylinder with piecewise power law nonhomogeneity due to asymmetric surface heating. Acta Mechanica, 225(10): 2903-2922. https://doi.org/10.1007/s00707-014-1204-3

[6] Tanigawa, Y., Ootao, Y., Kawamura, R. (1991). Thermal bending of laminated composite rectangular plates and nonhomogeneous plates due to partial heating. Journal of Thermal Stresses, 14(3): 285-308. https://doi.org/10.1080/01495739108927069

[7] Ootao, Y. (2011). Transient thermoelastic analysis for a multilayered hollow sphere with piecewise power law nonhomogeneity. Composite Structures, 93(7): 17171725. https://doi.org/10.1016/j.compstruct.2010.12.008

[8] Ootao, Y., Tanigawa, Y. (2006). Transient thermoelastic analysis for a functionally graded hollow cylinder. Journal of Thermal Stresses, 29(11): 1031-1046. https://doi.org/10.1080/01495730600710356

[9] Sladek, J., Sladek, V., Zhang, C. (2003). Transient heat conduction analysis in functionally graded materials by the meshless local boundary integral equation method. Computational Materials Science, 28(3-4): 494-504. https://doi.org/10.1016/j.commatsci.2003.08.006

[10] Wang, B.L., Mai, Y.W. (2005). Transient onedimensional heat conduction problems solved by finite element. International Journal of Mechanical Sciences, 47(2):

303-317. https://doi.org/10.1016/j.ijmecsci.2004.11.001

[11] Hosseini, S.M., Akhlaghi, M., Shakeri, M. (2008). Heat conduction and heat wave propagation in functionally graded thick hollow cylinder base on coupled thermoelasticity without energy dissipation. Heat and 
Mass Transfer, 44(12): 1477-1484. https://doi.org/10.1007/s00231-008-0381-9

[12] Matysiak, S.J. (1991). On certain problems of heat conduction in periodic composites. ZAMM-Journal of Applied Mathematics and Mechanics/Zeitschrift für Angewandte Mathematik und Mechanik, 71(12): 524528. https://doi.org/10.1002/zamm.19910711218

[13] Matysiak, S.J., Woźniak, C. (1987). On the modelling of heat conduction problem in laminated bodies. Acta Mechanica, 65(1): 223-238. https://doi.org/10.1007/BF01176884

[14] Woźniak, C., Wagrowska, M., Szlachetka, O. (2012). Asymptotic modelling and design of some microlayered functionally graded heat conductors. ZAMM - Journal of Applied Mathematics and Mechanics/Zeitschrift für Angewandte Mathematik und Mechanik, 92(10): 841848. https://doi.org/10.1002/zamm.201100092

[15] Matysiak, S.J., Perkowski, D.M. (2010). On heat conduction in a semi-infinite laminated layer. Comparative results for two approaches. International Communications in Heat and Mass Transfer, 37(4): 343 349.

[16] Perkowski, D.M., Kulchytsky-Zhyhailo, R., Kołodziejczyk, W. (2018). On axisymmetric heat conduction problem for multilayer graded coated halfspace. Journal of Theoretical and Applied Mechanics, 56(1): 147-156. https://doi.org/10.15632/jtampl.56.1.147

[17] Kulchytsky-Zhyhailo, R., Matysiak, S.J., Perkowski, D. M. (2011). Plane contact problems with frictional heating for a vertically layered half-space. International Journal of Heat and Mass Transfer, 54(9-10): 1805-1813. https://doi.org/10.1016/j.ijheatmasstransfer.2010.10.040

[18] Kulchytsky-Zhyhailo, R., Matysiak, S.J. (2005). On heat conduction problem in a semi-infinite periodically laminated layer. International Communications in Heat and Mass Transfer, 32(1-2): 123-132. https://doi.org/10.1016/j.icheatmasstransfer.2004.08.02 3

[19] Matysiak, S.J., Perkowski, D.M. (2011). Axially symmetric problems of heat conduction in a periodically laminated layer with vertical cylindrical hole. International Communications in Heat and Mass Transfer, 38(4): 410-417.

[20] Ganczarski, A., Szubartowski, D. (2020). Problems of thick functionally graded material structures under thermomechanical loadings. In Advances in Mechanics of High-Temperature Materials, pp.

$57-78$ https://doi.org/10.1007/978-3-030-23869-8_3

[21] Wang, H., Qin, Q.H., Kang, Y.L. (2006). A meshless model for transient heat conduction in functionally graded materials. Computational Mechanics, 38(1): 5160. https://doi.org/10.1007/s00466-005-0720-3

[22] Tanaka, M., Tanaka, K. (1980). Transient heat conduction problems in inhomogeneous media discretized by means of boundary-volume element. Nuclear Engineering and Design, 60(3): 381-387.

[23] Voigt, W. (1889). Ueber die Beziehung zwischen den beiden Elasticitätsconstanten isotroper Körper. Annalen der Physik, 274(12): 573-587. https://doi.org/10.1002/andp.18892741206

[24] Reuß, A. (1929). Berechnung der fließgrenze von mischkristallen auf grund der plastizitätsbedingung für einkristalle. ZAMM-Journal of Applied Mathematics and Mechanics/Zeitschrift für Angewandte Mathematik und Mechanik, 9(1): 49-58. https://doi.org/10.1002/zamm.19290090104

[25] Kaczyński, A., Matysiak, S.J. (1988). Plane contact problems for a periodic two-layered elastic composite. Ingenieur-Archiv, 58(2): https://doi.org/10.1007/BF00536233

\section{NOMENCLATURE}

$T$

$T^{\prime}$

$B i=\alpha R_{0} / K_{0}$

$\breve{K}(r)$

$R_{0}$

$R_{1}$

\section{Greek symbols}

$\alpha$

$\theta_{0}$

$\delta$

\section{Subscripts}

temperature deviation in the points of pipe form the temperature of outer medium, $\mathrm{K}$ the derivative of function $T$,

Biota number, thermal conductivity, W. $\mathrm{m}^{-1} \cdot \mathrm{K}^{-1}$ outer radius of pipe, $m$ inner radius of pipe, $\mathrm{m}$

\section{Greek symbols}

the coefficient of heat exchange between the outer surface of pipe and the outer medium value of temperature of outer medium, $\mathrm{K}$ dimensionless thickness of cells

indices related to sublayer numbering 